\section{Como a atenção primária à saúde pode fortalecer a alimentação adequada enquanto direito na América Latina?}

\author{
Fernanda Cangussu Botelho e Ivan \\ França Junior ${ }^{1}$
}

Como citar Botelho FC, França Junior I. Como a atenção primária à saúde pode fortalecer a alimentação adequada enquanto direito na América Latina? Rev Panam Salud Publica. 2018;42:e159. https://doi.org/10.26633/RPSP.2018.159

\section{RESUMO}

Há 40 anos, a Declaração de Alma-Ata reforçou a saúde como direito humano, apresentou a atenção primária à saúde (APS) como caminho para atingir um grau de saúde aceitável para todos e incorporou a questão alimentar e nutricional como parte integrante dos cuidados primários em saúde. O direito humano à alimentação adequada (DHAA) está intimamente relacionado com o direito à saúde, pois é requisito para a existência de condições de vida digna para promover a saúde. As particularidades históricas e a posição políticoeconômica da América Latina representam barreiras para a realização plena dos direitos humanos, e especialmente dos direitos sociais. Nesse sentido, o objetivo deste artigo é explorar os modos pelos quais os serviços de APS podem alavancar o DHAA na América Latina. Adicionalmente, são apresentadas medidas que exemplificam como os países podem fortalecer o DHAA a partir da APS. Finalmente, o texto se propõe a resgatar o potencial emancipatório da APS na América Latina vislumbrando sua atuação na realização de direitos humanos para além do direito à saúde. O panorama apresentado demonstra a capacidade de resposta da APS no sentido de efetivar direitos humanos interdependentes da saúde no contexto latino-americano.

\footnotetext{
Universidade de São Paulo (USP), Faculdade de Saúde Pública, Departamento de Saúde, Ciclos de Vida e Sociedade, São Paulo (SP), Brasil. Correspondência: Fernanda Cangussu Botelho, fer.cangussu@gmail.com
}

Palavras-chave Atenção primária à saúde; segurança alimentar e nutricional; direitos humanos.

Nos países latino-americanos, as reformas nos sistemas de saúde, a partir da década de 1990, foram fortemente influenciadas pela Declaração de Alma-Ata (1). Esse documento reforçou a saúde como um direito humano fundamental e apontou a atenção primária à saúde (APS) como caminho para atingir um grau de saúde aceitável para todos, com base na justiça social (2). Seguiu-se, na América Latina, o desafio de implementar modelos de APS sustentados por direitos humanos, participação social, empoderamento comunitário e articulação intersetorial (1).

$\mathrm{O}$ direito humano à alimentação adequada (DHAA) e o direito à saúde estão intimamente relacionados, já que constituem condições básicas para o bem-estar e a dignidade humana (3). Além disso, a violação do primeiro pode acarretar consequências ao bem-estar social e mental e o comprometimento do estado nutricional (4). Sendo assim, a Declaração de Alma-Ata incorporou a alimentação e a nutrição como elementos integrantes dos cuidados primários em saúde (2). Abordar a alimentação e a saúde como direitos é uma estratégia que tem se mostrado bem-sucedida na promoção da saúde, envolvendo a responsabilidade dos governos pelo monitoramento e efetivação desses direitos, participação social, priorização dos vulneráveis e não discriminação (3).

Pensar sobre o potencial da APS na garantia dos direitos a alimentação e saúde na América Latina implica sublinhar algumas especificidades. Diversos países latino-americanos passaram por ditaduras no período de 1960 a 1980, o que produziu entraves ao desenvolvimento dos sistemas de saúde na região (1). Nesses países, a implementação da APS coincidiu com os movimentos de redemocratização, que abriram espaço para o fortalecimento dos direitos e da participação social na saúde pública (1).

Além das semelhanças históricas e das desigualdades sociais que marcam a região, outro denominador comum na América Latina é a posição histórica de dependência e subordinação aos centros econômicos. Mesmo com governos democráticos, essa mediação política que prioriza as orientações macroeconômicas dos países hegemônicos compromete a garantia dos direitos humanos na América Latina, inclusive do DHAA e do direito à saúde (5). 
Partindo da relação entre esses dois direitos humanos, o objetivo da presente reflexão é explorar as possibilidades de atuação da APS para concretizar o DHAA. Pretendeu-se, ainda, propor um panorama do que pode ser feito, mas não necessariamente do que deveria estar sendo feito, já que este artigo pretende ser instigador e não prescritivo.

\section{RESGATE DO PERCURSO EM DIREÇÃO AO DIREITO HUMANO À ALIMENTAÇÃO ADEQUADA}

A alimentação foi reconhecida como direito na Declaração Universal dos Direitos Humanos (6). Nesse documento, a alimentação é apresentada como um dos elementos para atingir a saúde e o bem-estar. $\mathrm{O}$ Pacto Internacional dos Direitos Econômicos, Sociais e Culturais (PIDESC) também reconhece o direito de estar livre da fome (7). A maioria dos países latino-americanos ratificou o PIDESC - sendo que a ratificação significa compromisso legal de efetivar esses direitos. Vale notar que Cuba apenas assinou, sem ratificar, esse documento.

Ao ratificar o PIDESC, os Estados-parte devem enviar relatórios periódicos ao Conselho Econômico e Social (CES), um dos seis órgãos principais da Organização das Nações Unidas (ONU), sobre as medidas tomadas para sua efetivação. O CES, através do seu Comitê dos Direitos Econômicos, Sociais e Culturais (composto por 18 auditores independentes), publica uma "lista de questões", que é respondida pelos Estados-parte. Para finalizar o ciclo, o CES publica suas conclusões sobre as questões.

Além disso, de forma mais elucidativa, a ONU produziu um comentário que detalha as obrigações dos Estados-parte em relação ao DHAA (8). A primeira obrigação é a de respeitar esse direito, ou seja: o próprio Estado-parte não deverá violar o DHAA. A obrigação de proteger envolve a garantia, pelo Estadoparte, de que terceiros, como empresas privadas ou indivíduos, não violem o DHAA. Já a obrigação de efetivar se divide em facilitar e prover: o Estado-parte facilita o DHAA quando fortalece o acesso e a utilização dos recursos e dos meios para garantir o modo de vida das pessoas; quando não houver outra possibilidade, o Estado precisa prover o DHAA diretamente.

\section{CARACTERIZAÇÃO DO DIREITO À SAÚDE}

O direito à saúde também está presente tanto na Declaração Universal dos Direitos Humanos (6) quanto no PIDESC, que reconhece o "direito de toda pessoa de desfrutar o mais elevado nível possível de saúde física e mental" (7, Art. 12).

No Comentário Geral n ${ }^{\circ} 14$, elaborado pela ONU, esse direito é descrito como contemplando aspectos biológicos individuais, condições socioeconômicas e recursos disponíveis em cada Estado-parte (9). O direito à saúde é abordado como direito de usufruir das instituições, bens, serviços e condições necessários para sua realização. São apresentados quatro elementos essenciais e inter-relacionados para sua concretização: disponibilidade, acessibilidade, aceitabilidade e qualidade.

A disponibilidade se concretiza quando os serviços de saúde pública e de cuidado em saúde, programas e bens de saúde, existem em quantidade suficiente dentro do Estado-parte. $\mathrm{O}$ item acessibilidade, por sua vez, apresenta quatro dimensões. A primeira se refere à não discriminação de qualquer natureza no acesso aos recursos existentes. A segunda é a acessibilidade física, especialmente para vulneráveis e marginalizados. A terceira é a acessibilidade econômica, ou seja, quando exigido, o pagamento pelos serviços de saúde deve ser viável para todos. A quarta dimensão trata do direito ao acesso a informações de saúde. O elemento de aceitabilidade se concretiza quando os serviços de saúde são ética e culturalmente apropriados. Isso inclui respeito e atenção especial a grupos minoritários, necessidades dos cidadãos nos diferentes ciclos de vida e sensibilidade às questões de gênero. Finalmente, a qualidade também implica serviços de saúde culturalmente apropriados e embasados cientificamente, além da disponibilidade de profissionais de saúde qualificados e dos equipamentos necessários ao cuidado (9).

\section{POSSIBILIDADES DE ABORDAGEM DO DIREITO HUMANO À ALIMENTAÇÃO ADEQUADA NA ATENÇÃO PRIMÁRIA À SAÚDE}

Conforme a Declaração de Alma-Ata, a APS constitui o primeiro nível de contato do indivíduo, da família e da comunidade com o sistema de saúde. Deve viabilizar cuidados baseados em métodos e tecnologias práticas, respaldados cientificamente e socialmente aceitáveis para garantir a promoção da saúde e a prevenção e controle de doenças (2).

No entanto, a implementação da APS não é uníssona, estando permeada por particularidades locais. Na América do Sul, por exemplo, predominam dois modelos de organização da APS: um caracterizado pela fragmentação e orientação do mercado e outro com domínio de intervenções públicas e territorialização (10). Levando em conta a posição da APS nos sistemas de saúde e os princípios propostos na Declaração de Alma-Ata, segue-se uma reflexão sobre como a APS pode respeitar, proteger e efetivar o DHAA. Algumas medidas práticas que exemplificam modos de concretizar o DHAA foram sumarizadas na figura 1.

\section{Obrigação de respeitar}

A obrigação do Estado-parte de respeitar o DHAA permeia todas as suas esferas, inclusive a APS. Pensando em disponibilidade, se não existem serviços de saúde para atender as demandas relacionadas à alimentação por exemplo, orientações sobre qualidade dos alimentos, efeitos dos alimentos na saúde e as melhores formas de prepará-los - em quantidade compatível com as 
FIGURA 1. Síntese de medidas para fortalecer o direito humano à alimentação adequada na atenção primária à saúde

PRESSUPOSTOS (transversais ao respeito, proteção e efetivação)

- Criar arcabouço legal para o reconhecimento do direito humano à alimentação adequada e do direito à saúde (priorizando inclusão na Constituição).

- Estabelecer políticas e planos de ação para efetivar o direito humano à alimentação adequada e o direito à saúde. - Criar garantias orçamentárias para implementação das políticas e planos de ação.

\section{RESPEITAR}

- Ampliar oferta de serviços de APS com capacidade de atender demandas de alimentação e nutrição.

- Priorizar a disponibilidade e 0 acesso de serviços de APS para populações vulneráveis.

- Eliminar taxas de pagamento por serviço na APS.

- Implementar programas e ações com embasamento científico.

- Garantir acesso de todos os grupos a esses programas e ações.

- Capacitar os profissionais de saúde atuantes e formar novos profissionais de saúde com base no respeito a práticas, normas e valores culturais, religiosos e éticos ligados à alimentação.

- Alocar recursos para ampliar a disponibilidade e acesso aos serviços de APS e para a capacitar e formar profissionais visando o respeito ao direito humano à alimentação adequada.

\section{PROTEGER}

- Criar/fortalecer sistemas de vigilância alimentar e nutricional.

- Incluir instrumentos de avaliação da insegurança alimentar que monitorem 0 acesso, a disponibilidade, a qualidade e a aceitabilidade dos alimentos.

- Priorizar mecanismos de identificação de violações do direito humano à alimentação adequada em populações vulneráveis.

- Promover articulação intersetorial, notadamente com a assistência social.

- Criar/fortalecer organismos de defesa dos direitos dos cidadãos

- Estabelecer parcerias com setores da sociedade civil atuantes na defesa dos direitos humanos.

- Criar mecanismos de exigibilidade do direito humano à alimentação adequada.

- Destinar tempo de trabalho dos profissionais de saúde para ações de vigilância alimentar e nutricional e demais de identificação de violações do direito humano à alimentação adequada.

- Capacitar os profissionais de saúde atuantes e formar novos profissionais de saúde para identificar situações de violação do direito humano à alimentação adequada.

- Alocar recursos para capacitação e formação profissional visando a proteção do direito humano à alimentação adequada.

\section{EFETIVAR}

- Priorizar abordagens educativas críticas e emancipatórias na abordagem da alimentação.

- Incluir discussões sobre questões sociais, pobreza e direitos na abordagem dos problemas alimentares.

- Criar estratégias para superar barreiras culturais, sociais e linguísticas entre profissionais de saúde e usuários.

- Promover ações voltadas para o diálogo entre profissionais de saúde e usuários.

- Dispor de espaços físicos e condições materiais para realização de ações educativas.

- Disponibilizar materiais educativos para os serviços de APS.

- Destinar tempo de trabalho dos profissionais de saúde para o planejamento de ações educativas.

- Inserir profissionais qualificados para abordar a alimentação nos serviços de APS, como nutricionistas.

- Capacitar os profissionais de saúde atuantes e formar novos profissionais de saúde para promover 0 direito humano à alimentação adequada.

- Alocar recursos para implementação de ações educativas e para a capacitação e formação profissional visando à efetivação do direito humano à alimentação adequada. necessidades da população, há desrespeito ao DHAA. Os países latino-americanos avançaram de forma distinta na questão da suficiência de serviços. Para a ONU, ainda restam problemas importantes nesse quesito, por exemplo, no México, Colômbia, Peru, Equador e Guatemala, sendo sugerida a alocação de recursos com prioridade para populações vulneráveis. Esta informação consta nas conclusões dos relatórios periódicos mais recentes emitidos pelo CES para cada um desse países: México (2018); Colômbia (2017); Peru (2012); Equador (2012); e Guatemala (2014) (11).

A mera existência de serviços de saúde não garante a presença de acessibilidade em todas as dimensões. Destaca-se o componente econômico, uma vez que, na visão dos usuários, o pagamento de taxas na APS resulta em menor uso dos serviços e no comprometimento da segurança alimentar, da estabilidade financeira e da atuação feminina na tomada de decisões em saúde (12), ou seja, uma miríade de violações de direitos humanos.

Os países sul-americanos que adotaram métodos de cobertura em saúde que não dependem de pagamento estão mais próximos da abordagem abrangente proposta pela Declaração de Alma-Ata, considerando elementos como a concepção de APS, o modo de organização dos serviços, a articulação intersetorial e a participação social (10). O Chile, por exemplo, desde a década de 1990, ampliou o investimento público na APS, visando a eliminar taxas para os beneficiários do Fundo Nacional de Saúde (1). Adicionalmente, o reconhecimento legal do direito à saúde tem se mostrado uma etapa importante para avançar na cobertura de saúde na América Latina (1).

Quanto à aceitabilidade, os serviços de APS respeitam o DHAA quando não infringem práticas, normas e valores culturais, religiosos e éticos ligados à alimentação. Isso pode parecer óbvio e deve estar dentro dos códigos de conduta dos profissionais de saúde. No entanto, no manejo da desnutrição infantil em Buenos Aires, por exemplo, há relatos de imposição da visão biomédica por profissionais de saúde, desconsiderando as concepções culturais das mães sobre a doença e forçando condutas (13). Assim, permanece o desafio de banir práticas que desrespeitam os direitos 
humanos, e ainda mais de fortalecer o potencial emancipatório e empoderador da APS.

No tocante à qualidade, destaca-se a dimensão de respaldo científico. Em 2005, o Brasil iniciou um programa de suplementação de ferro para gestantes via APS com base em evidências científicas e recomendações da Organização Mundial da Saúde (OMS). No entanto, a sua implementação enfrentou impasses em nível local por baixa cobertura e distribuição desigual de suplementos entre as mulheres (14). Quando o serviço de saúde não promove um cuidado pautado em evidências científicas para todos, há desrespeito do DHAA por baixa qualidade. O mesmo acontece quando o serviço realiza práticas já reportadas cientificamente como inapropriadas, por exemplo, ao orientar introdução de alimentação complementar em crianças menores que 6 meses através de chás e sucos.

\section{Obrigação de proteger}

A APS tem grande potencial para proteger o DHAA, principalmente na identificação de violações desse direito por terceiros. A matéria-prima da APS nesse caso está na geração de informações dentro do território.

Considerando a acessibilidade e a disponibilidade, a APS protege o DHAA quando dispõe de mecanismos para detectar situações de insegurança alimentar e os utiliza sem discriminação. Da mesma forma que a APS pode monitorar, via vigilância em saúde, doenças infecciosas ou a violência contra crianças e adolescentes, por exemplo, pode monitorar o estado de efetivação do DHAA, via vigilância alimentar e nutricional. Desde 1977, Cuba, por exemplo, possui um sistema de vigilância nutricional para gerar informações sobre o estado nutricional e o acesso à alimentação adequada pela população (15).

A APS também pode atuar na identificação de situações de negligência alimentar em ambientes institucionais (por exemplo, creches, escolas e instituições de longa permanência) e na família. Ressalta-se também que as ferramentas de identificação de insegurança alimentar revelam diferentes faces de violações do DHAA. Portanto, instrumentos como escalas psicométricas de avaliação da insegurança alimentar, como a US Household Food Security Survey Measure e suas derivadas, são mais interessantes do que somente o diagnóstico de desfechos biológicos, como a desnutrição (4).

A fome apresenta um caráter vexatório para aqueles que a vivenciam. Nesse sentido, o elemento de aceitabilidade inclui a confidencialidade ao reportar informações sobre violações do DHAA no território às autoridades competentes. A atuação diante desses casos demanda articulação intersetorial, notadamente com a assistência social, para recorrer a programas existentes, como os de transferência de renda. Persistindo as violações, é possível acionar instituições de defesa dos direitos dos cidadãos, como o Ministério Público, especialmente nos países com arcabouço legal para o DHAA. Nos países com legislações menos avançadas, as parcerias com organizações de defesa do
DHAA (por exemplo, Food Information and Action Network, ou FIAN International, https://www.fian. org/) são um caminho.

Para que a proteção do DHAA seja factível, destaca-se a importância de recursos humanos qualificados e condições estruturais na APS, que são componentes da qualidade dos serviços. Na perspectiva dos médicos da APS, é necessário treinamento para abordar a segurança alimentar, tanto em aspectos técnicos, quanto para não gerar constrangimentos aos usuários (16). A destinação de tempo de trabalho para a execução dessas tarefas é imprescindível para que não se tornem mais uma atribuição impraticável na rotina de trabalho.

\section{Obrigação de efetivar}

Dentro das duas ações que compõem a efetivação do DHAA, a APS é mais um espaço para facilitação do que para o provimento, embora historicamente tenha sediado experiências de distribuição de alimentos na América Latina. A efetivação de um direito depende de muitas esferas, incluindo os respaldos legislativo, administrativo e financeiro. Cabe à APS a promoção desse direito, fortalecendo as condições para que ele se realize, principalmente através do conhecimento sobre o tema.

Ações educativas na APS são centrais para a facilitação do DHAA. Em especial, a educação alimentar e nutricional pode sensibilizar os usuários quanto à alimentação como direito e quanto à problematização da pobreza, da fome, do consumo de alimentos de baixa qualidade e das desigualdades sociais e econômicas geradoras dessas condições. Contudo, essa educação deve contemplar o diálogo entre profissionais e usuários e visar à autonomia do sujeito (17).

No que se refere a disponibilidade e acessibilidade, a APS facilita o DHAA quando realiza ações de promoção da alimentação adequada na perspectiva dos direitos humanos, dentro da unidade de saúde e em equipamentos do território acessíveis, sem qualquer tipo de discriminação. As hortas nos serviços de APS, por exemplo, reforçam a ação comunitária, promovem habilidades e resgatam práticas e hábitos tradicionais (18), ou seja, desfechos que coadunam com o DHAA.

Para a aceitabilidade, as ações devem valorizar a cultura e as preferências alimentares dos usuários e pautar-se por princípios éticos. De forma geral, diferenças culturais e religiosas relacionadas à alimentação podem ser barreiras para o enfrentamento de questões alimentares. O quadro teórico proposto por Santos (19) pode auxiliar ações de facilitação do DHAA na APS. O autor propõe uma concepção multicultural de direitos humanos, partindo do reconhecimento da incompletude de todas as culturas para, assim, manter um diálogo intercultural, evitando a imposição de uma cultura sobre a outra (19). O caso dos imigrantes bolivianos no município de São Paulo é um exemplo. Cursos de espanhol e sobre cultura boliviana para os profissionais de saúde brasileiros e contratação de agentes comunitários de saúde (ACS) bolivianos foram 
propostas que procuraram aproximar os imigrantes dos serviços de APS (20).

Promover a alimentação adequada na perspectiva dos direitos humanos pode ser uma tarefa difícil. A inserção de profissionais de saúde qualificados, a educação permanente, as condições dignas de trabalho e o provimento adequado de materiais promovem a qualidade das ações de facilitação do DHAA. Os Núcleos de Apoio à Saúde da Família (NASF) no Brasil são um exemplo de estratégia para ampliar o escopo e a resolutividade da APS, inserindo profissionais, inclusive nutricionistas, além da equipe mínima. O nutricionista era o terceiro profissional mais presente nas equipes de NASF 3 anos após a proposição do programa (21), aumentando as oportunidades de facilitação do DHAA na APS.

\section{CONSIDERAÇÕES FINAIS}

Esta apresentação oferece uma reflexão sobre como ampliar a capacidade de resposta da APS frente aos problemas alimentares vivenciados pela população latino-americana. A APS não é apenas um cenário de identificação de violações do DHAA, é também um espaço de atuação em prol da efetivação de direitos humanos para além do direito à saúde, visando à superação das desigualdades. As medidas apresentadas na figura 1 não esgotam todas as possibilidades para essa atuação, mas subsidiam reflexões a serem feitas em cada país considerando a conformação da APS localmente. Ainda que se direcionem mais aos formuladores de políticas, podem ser usadas como instrumento para o ativismo pelo tema, já que direitos são conquistados a partir de participação e engajamento social.

A presente reflexão, 40 anos após a Declaração de Alma-Ata, chama a APS para repensar as práticas de saúde na perspectiva dos direitos humanos, resgatando o potencial emancipatório da APS na América Latina. A APS não pode, sozinha, solucionar os problemas da região, mas sua atuação pode ser ampliada para promover condições de vida digna e, consequentemente, saúde plena para a população.

Agradecimentos. Ao Conselho Nacional de Desenvolvimento Científico e Tecnológico (CNPq) por concessão de bolsa de mestrado à primeira autora e bolsa de produtividade em pesquisa ao segundo autor.

Conflitos de interesse. Nada declarado pelos autores.

Declaração. As opiniões expressas no manuscrito são de responsabilidade exclusiva dos autores e não refletem necessariamente a opinião ou política da RPSP / PAJPH ou da Organização Pan-Americana da Saúde (OPAS).

\section{REFERÊNCIAS}

1. Atun R, Andrade LOM, Almeida G, Cotlear D, Dmytraczenko T, Frenz P, et al. Health-system reform and universal health coverage in Latin America. Lancet. 2015;385:1230-47.

2. Organização Mundial da Saúde (OMS). Declaração de Alma-Ata. Conferência Internacional sobre Cuidados Primários em Saúde. Alma-Ata; 1987. Disponível em: www.who.int/publications/almaata_declaration_en.pdf?ua $=1 \& u a=1$ Acessado em agosto de 2018.

3. Chilton M, Rose D. A rights-based approach to food insecurity in the United States. Am J Public Health. 2009;99(7):1203-11.

4. Kepple AW, Segall-Corrêa AM. Conceituando e medindo segurança alimentar e nutricional. Cienc Saude Colet. 2011;16(1):187-99.

5. Netto JP. Democracia e direitos humanos na América Latina: aportes necessários ao debate. Em: Freire SM. Direitos humanos e questão social na América Latina. Rio de Janeiro: Gramma; 2009. Pp. 3-12.

6. United Nations. Universal Declaration of Human Rights. Paris: United Nations General Assembly; 1948. Disponível em: http://www.un.org/ en/universal-declaration-human-rights/ Acessado em agosto de 2018.
7. Brasil. Decreto 591/1992. Atos Internacionais. Pacto Internacional sobre Direitos Econômicos, Sociais e Culturais. Promulgação. Disponível em: http://www.planalto.gov.br/ ccivil_03/decreto/1990-1994/d0591. htm Acessado em agosto de 2018.

8. United Nations, Committee on Economic, Social and Cultural Rights (CESCR/UN). General Comment no 12: the right to adequate food. Genebra: United Nations; 1999. Disponível em: http://www.refworld.org/pdfid/4538838c11.pdf Acessado em agosto de 2018.

9. United Nations, Committee on Economic, Social and Cultural Rights (CESCR/UN). General Comment no 14: The Right to the Highest Attainable Standard of Health. Genebra; United Nations; 2000. Disponível em: http://www.refworld.org/pdfid / 4538838d0.pdf Acessado em agosto de 2018.

10. Ramírez NA, Giovanella L, Romero RV, Silvad HT, Almeida PT, Ríos G, et al. Mapping primary health care renewal in South America. J Fam Pract. 2016;33(3):261-7.

11. United Nations. Office of the High Commissioner for Human Rights. Concluding observations. Disponível em: https://tbinternet.ohchr.org/_ layouts / treatybodyexternal/ TBSearch.aspx? Lang=en\&Treaty ID=9\&DocTypeID=5 Acessado em agosto de 2018.

12. Johnson A, Goss A, Beckerman J, Castro A. Hidden costs: The direct and indirect impact of user fees on access to malaria treatment and primary care in Mali. Soc Sci Med. 2012;75:1786-92.

13. Herkovitz D. Las fronteras de la medicalización: tensiones en torno a la identificación y valoración de la desnutrición infantil en un centro de atención primaria de la ciudad de Buenos Aires. Cienc Saude Colet. 2012;17(9):2543-51.

14. Linhares AO, Linhares RdaS, Cesar JA. Iniquidade na suplementação de sulfato ferroso entre gestantes no sul do Brasil. Rev Bras Epidemiol. 2017;20:650-60.

15. Rodríguez SO; García AMN. Estado de la nutrición materno-infantil en la provincia de Camagüey. Rev Cubana Aliment Nutr. 2013;23(2): 284-96.

16. Palakshappa D, Vasan A, Khan S, Seifu L, Feudtner C, Fiks AG. Clinicians' perceptions of screening for food insecurity in suburban pediatric practice. Pediatrics. 2017; 140(1):1-9. 
17. Santos LAS. O fazer educação alimentar e nutricional: algumas contribuições para reflexão. Cienc Saude Colet. 2012;17(2):453-62.

18. Costa CGA, Garcia MT, Ribeiro SM, Salandini MFS, Bógus CM. Hortas comunitárias como atividade promotora de saúde: uma experiência em Unidades Básicas de Saúde. Cienc Saude Coletiva. 2015;20(10): 3099-110.
19. Santos BS. Por uma concepção multicultural de direitos humanos. Rev Crit Cienc Sociais. 1997:48:11-32.

20. Martes ACB, Faleiros SM. Acesso dos imigrantes bolivianos aos serviços públicos de saúde na cidade de São Paulo. Saude Soc. 2013;22:351-64.

21. Patrocínio SSDSM, Machado CV, Fausto MCR. Núcleo de Apoio à Saúde da Família: proposta nacional e implementação em municípios do Rio de Janeiro. Saude Debate. 2015;39:105-19.

Manuscrito recebido em 14 de janeiro de 2018. Aceito em versão revisada em 7 de agosto de 2018.
ABSTRACT

\section{Can primary health care strengthen the right to adequate food as a human right in Latin America?}

Keywords Primary health care; food and nutrition security; human rights.
Forty years ago, the Declaration of Alma-Ata emphasized health as a human right, introduced primary health care (PHC) as a strategy to attain an acceptable level of health for all, and included the issue of food and nutrition as an integral part of PHC. The right to adequate food (RAF) is closely related to the right to health, since it is essential to ensure dignified living conditions that promote health. The historical peculiarities and the political and economic position of Latin America constitute barriers for the full realization of human rights, and especially social rights. In this sense, the present article aims to explore the modes by which PHC services can leverage the RAF in Latin America. In addition, the article describes measures that exemplify how countries can strengthen RAF through PHC. Finally, the text seeks to recover the emancipatory potential of PHC through a vision of human rights enforcement beyond the right to health. The overview shows that PHC has the capacity to fulfill human rights that are interdependent on health in the Latin American context.
RESUMEN

¿Cómo la atención primaria de salud puede fortalecer la alimentación adecuada como derecho en América Latina?

Palabras clave
Hace 40 años, la Declaración de Alma-Ata reforzó la salud como derecho humano, presentó la atención primaria de salud (APS) como camino para alcanzar un grado de salud aceptable para todos e incorporó la alimentación y nutrición como parte de los cuidados primarios en salud. El derecho humano a la alimentación adecuada (DHAA) está íntimamente relacionado con el derecho a la salud, pues es un requisito indispensabel para la existencia de condiciones dignas para promover la salud. Las particularidades históricas y la posición político-económica de América Latina representan barreras para la promoción plena de los derechos humanos, y especialmente de los derechos sociales. En este sentido, el objetivo de este artículo es explorar la manera como los servicios de APS pueden impulsar el DHAA en América Latina. Adicionalmente, se presentan medidas que ejemplifican cómo los países pueden fortalecer el DHAA a partir de la APS. Finalmente, el texto se propone rescatar el potencial emancipatorio de la APS en América Latina vislumbrando su actuación en la promoción de derechos humanos más allá del derecho a la salud. El panorama presentado demuestra la capacidad de respuesta de la APS para hacer efectivos los derechos humanos interdependientes de la salud en el contexto latinoamericano.

Atención primaria de salud; seguridad alimentaria y nutricional; derechos humanos. 